\title{
Optimal Backward Perturbation Analysis for the Block Skew Circulant Linear Systems with Skew Circulant Blocks
}

\author{
Zhaolin Jiang, ${ }^{1}$ Juan Li, ${ }^{1,2}$ and Jianwei Zhou ${ }^{1}$ \\ ${ }^{1}$ Department of Mathematics, Linyi University, Linyi, Shandong 276000, China \\ ${ }^{2}$ Department of Mathematics, Shandong Normal University, Jinan, Shandong 250014, China
}

Correspondence should be addressed to Juan Li; zxc123pollijuan@163.com

Received 27 February 2014; Revised 24 April 2014; Accepted 24 May 2014; Published 15 June 2014

Academic Editor: Ahmet Yasar Ozban

Copyright (c) 2014 Zhaolin Jiang et al. This is an open access article distributed under the Creative Commons Attribution License, which permits unrestricted use, distribution, and reproduction in any medium, provided the original work is properly cited.

We first give the block style spectral decomposition of arbitrary block skew circulant matrix with skew circulant blocks. Secondly, we obtain the singular value of block skew circulant matrix with skew circulant blocks as well. Finally, based on the block style spectral decomposition, we deal with the optimal backward perturbation analysis for the block skew circulant linear system with skew circulant blocks.

\section{Introduction}

A block skew circulant matrix with skew circulant blocks with the first row $\left(a_{11}, \ldots, a_{1 m}, a_{21}, \ldots, a_{2 m}, \ldots, a_{n 1}, \ldots, a_{n m}\right)$ is meant a square matrix of the following form:

$$
\left(\begin{array}{ccccc}
A_{1} & A_{2} & \cdots & A_{n-1} & A_{n} \\
-A_{n} & A_{1} & A_{2} & \cdots & A_{n-1} \\
\vdots & \ddots & \ddots & \ddots & \vdots \\
-A_{3} & \cdots & -A_{n} & A_{1} & A_{2} \\
-A_{2} & -A_{3} & \cdots & -A_{n} & A_{1}
\end{array}\right)
$$

and for any $k=1,2, \ldots, n$,

$$
A_{k}=\left(\begin{array}{ccccc}
a_{k 1} & a_{k 2} & \cdots & a_{k(m-1)} & a_{k m} \\
-a_{k m} & a_{k 1} & a_{k 2} & \cdots & a_{k(m-1)} \\
\vdots & \ddots & \ddots & \ddots & \vdots \\
-a_{k 3} & \cdots & -a_{k m} & a_{k 1} & a_{k 2} \\
-a_{k 2} & -a_{k 3} & \cdots & -a_{k m} & a_{k 1}
\end{array}\right),
$$

denoted by $\mathrm{BC}_{-1,-1}^{n, m}\left(a_{11}, \ldots, a_{1 m}, \ldots, a_{n 1}, \ldots, a_{n m}\right)$. Note that in this paper all facts are based on real field.

Skew circulant matrices have important applications in various disciplines including image processing, signal processing, solving Toeplitz matrix problems, preconditioner, and solving least squares problems in [1-10].
Liu and Guo [11] gave the optimal backward perturbation analysis for a block circulant linear system. Li et al. [12] gave the style spectral decomposition of skew circulant matrix firstly and then dealt with the optimal backward perturbation analysis for the skew circulant linear system. The optimal backward perturbation bounds for underdetermined systems are studied by J.G. Sun and Z. Sun in [13]. Some new theorems generalizing a result of Oettli and Prager are applied to a posteriori analysis of the compatibility of a computed solution to the uncertain data of a linear system by Rigal and Gaches in [14]. Systems with BC structure appear in the context of multichannel signal estimation $[15,16]$, image restoration [17], cyclic convolution filter banks [18], texture synthesis and recognition [19], and so on.

The block skew circulant matrix with skew circulant blocks is an extension of skew circulant matrix and we believe the block skew circulant linear system with skew circulant blocks can be used in those fields as well. In this paper, firstly, by using the style spectral decomposition of a special skew circulant matrix $C$ in [12], we get the block style spectral decomposition of arbitrary block skew circulant matrix with skew circulant blocks. Secondly, we obtain the singular value of block skew circulant matrix with skew circulant blocks as well. Finally, we deal with the optimal backward perturbation analysis for the block skew circulant linear system with skew circulant blocks on the base of its block style spectral decomposition. 


\section{The Block Style Spectral Decomposition of Block Skew Circulant Matrix with Skew Circulant Blocks}

Let matrix $A$ be a block skew circulant matrix with skew circulant blocks as in the form of (1); then by using the properties of Kronecker products in [20], the matrix $A$ can be decomposed as

$$
A=\sum_{k=1}^{n}\left(C_{n \times n}^{k-1} \otimes A_{k}\right)
$$

where

$$
C_{n \times n}=\left(\begin{array}{ccccc}
0 & 1 & 0 & \cdots & 0 \\
0 & 0 & 1 & \cdots & 0 \\
\vdots & \ddots & \ddots & \ddots & \vdots \\
0 & \cdots & 0 & 0 & 1 \\
-1 & 0 & \cdots & 0 & 0
\end{array}\right)_{n \times n}
$$

According to the style spectral decomposition of basic skew circulant (please refer to equations (10) and (11) in [12]), the style spectral decomposition of the matrix $C_{n \times n}^{k}$ is

$$
C_{n \times n}^{k}=Q C_{0}^{k} Q^{T}
$$

where $Q$ is an orthogonal matrix, $\theta_{j}=((2 j-1) / n) \pi$,

$$
\begin{aligned}
& C_{0}^{k}=\left(\begin{array}{cccc}
C_{1}^{k} & & & \\
& C_{2}^{k} & & \\
& & \ddots & \\
& & & C_{n / 2}^{k}
\end{array}\right), \quad(n \text { is even }), \\
& C_{0}^{k}=\left(\begin{array}{cccc}
C_{1}^{k} & & & \\
& \ddots & & \\
& & C_{(n-1) / 2}^{k} & \\
& & & (-1)^{k}
\end{array}\right), \quad(n \text { is odd }), \\
& C_{j}^{k}=\left(\begin{array}{cc}
\cos k \theta_{j} & \sin k \theta_{j} \\
-\sin k \theta_{j} & \cos k \theta_{j}
\end{array}\right), \\
& j= \begin{cases}1,2, \ldots, \frac{n}{2}, & \text { when } n \text { is even, } \\
1,2, \ldots, \frac{n-1}{2}, & \text { when } n \text { is odd. }\end{cases}
\end{aligned}
$$

Consider (3) and (5); the matrix $A$ can be decomposed as

$$
\begin{aligned}
A & =\sum_{k=1}^{n}\left(C_{n \times n}^{k-1} \otimes A_{k}\right)=\sum_{k=1}^{n}\left(Q C_{0}^{k-1} Q^{T}\right) \otimes A_{k} \\
& =\sum_{k=1}^{n}\left(Q \otimes I_{m}\right)\left(C_{0}^{k-1} \otimes A_{k}\right)\left(Q^{T} \otimes I_{m}\right) \\
& =\left(Q \otimes I_{m}\right)\left(\sum_{k=1}^{n} C_{0}^{k-1} \otimes A_{k}\right)\left(Q^{T} \otimes I_{m}\right) .
\end{aligned}
$$

Noticing that $Q \otimes I_{m}$ is an orthogonal matrix, so (8) is the block style spectral decomposition of the matrix $A$.

\section{The Structured Perturbation Analysis}

In this section, we give the structured perturbation analysis for the block skew circulant linear systems with skew circulant blocks.

3.1. Condition Number and Relative Error of Block Skew Circulant Linear System with Skew Circulant Blocks. Consider

$$
A x=b,
$$

where $A$ is defined in (1).

From (8) and the property of Kronecker products in [20], we express the matrix $A$ by using the elements in its first row as

$$
\begin{aligned}
A & =\sum_{k=1}^{n}\left(C_{n \times n}^{k-1} \otimes A_{k}\right) \\
& =\sum_{k=1}^{n}\left[C_{n \times n}^{k-1} \otimes\left(\sum_{l=1}^{m} a_{k l} C_{m \times m}^{l-1}\right)\right] \\
& =\sum_{k=1}^{n} \sum_{l=1}^{m} a_{k l}\left(C_{n \times n}^{k-1} \otimes C_{m \times m}^{l-1}\right),
\end{aligned}
$$

where

$$
C_{m \times m}=\left(\begin{array}{ccccc}
0 & 1 & 0 & \cdots & 0 \\
0 & 0 & 1 & \cdots & 0 \\
\vdots & \ddots & \ddots & \ddots & \vdots \\
0 & \cdots & 0 & 0 & 1 \\
-1 & 0 & \cdots & 0 & 0
\end{array}\right)_{m \times m}
$$

We denote the eigenvalues of matrix $C_{n \times n}$ as $\varepsilon_{i}(i=$ $1,2, \ldots, n)$, and denote the eigenvalues of matrix $C_{m \times m}$ as $\delta_{j}$ $(j=1,2, \ldots, m)$; then the eigenvalues of $A$ are (regarding more properties, please refer to $[20,21]$ )

$$
\lambda_{i j}=\sum_{k=1}^{n} \sum_{l=1}^{m} a_{k l} \varepsilon_{i}^{k-1} \delta_{j}^{l-1} .
$$

Lemma 1. $A$ is an invertible matrix if and only if $f\left(\varepsilon_{i}, \delta_{j}\right) \neq 0$ $(i=1,2, \ldots, n, j=1,2, \ldots, m)$, where

$$
f\left(\varepsilon_{i}, \delta_{j}\right)=\lambda_{i j}=\sum_{k=1}^{n} \sum_{l=1}^{m} a_{k l} \varepsilon_{i}^{k-1} \delta_{j}^{l-1} .
$$

Let

$$
\begin{gathered}
\sigma_{i j}=\left|f\left(\varepsilon_{i}, \delta_{j}\right)\right|, \quad i=1,2, \ldots, n, j=1,2, \ldots, m, \\
\mathscr{K}=\frac{\max \left\{\sigma_{i j}\right\}}{\min \left\{\sigma_{i j}\right\}} .
\end{gathered}
$$

Theorem 2. If $A=B C_{-1,-1}^{n, m}\left(a_{11}, \ldots, a_{1 m}, \ldots, a_{n 1}, \ldots, a_{n m}\right)$ is a block skew circulant matrix with skew circulant blocks, then the singular values of matrix $A$ are $\sigma_{11}, \ldots, \sigma_{1 m}, \sigma_{21}, \ldots, \sigma_{2 m}, \ldots$, $\sigma_{n 1}, \ldots, \sigma_{n m}$. 
Proof. Obviously, the matrix $A$ has a form as (1) and the conjugate transpose of $A$ is

$$
A^{*}=\left(\begin{array}{ccccc}
A_{1}^{*} & -A_{n}^{*} & \cdots & -A_{3}^{*} & -A_{2}^{*} \\
A_{2}^{*} & A_{1}^{*} & \ddots & \vdots & -A_{3}^{*} \\
\vdots & A_{2}^{*} & \ddots & -A_{n}^{*} & \vdots \\
A_{n-1}^{*} & \vdots & \ddots & A_{1}^{*} & -A_{n}^{*} \\
A_{n}^{*} & A_{n-1}^{*} & \cdots & A_{2}^{*} & A_{1}^{*}
\end{array}\right) .
$$

Through a direct calculation, we can get $A A^{*}=A^{*} A$, and that means that $A$ is a normal matrix. By using Theorem 2.5.4 in [22], we know that $A$ is unitarily diagonalizable. That is, there is a unitary matrix $\mathbb{U} \in M_{m n}$ such that

$$
\mathbb{U}^{*} A \mathbb{U}=\Lambda=\operatorname{diag}\left(\lambda_{11}, \ldots, \lambda_{1 m}, \ldots, \lambda_{n 1}, \ldots, \lambda_{n m}\right) \text {. }
$$

where $\lambda_{i j}(i=1,2, \ldots, n, j=1,2, \ldots, m)$ are the eigenvalues of $A$. Taking a conjugate transpose at both sides of (16),

$$
\mathbb{U}^{*} A^{*} \mathbb{U}=\Lambda^{*}=\operatorname{diag}\left(\bar{\lambda}_{11}, \ldots, \bar{\lambda}_{1 m}, \ldots, \bar{\lambda}_{n 1}, \ldots, \bar{\lambda}_{n m}\right),
$$

and so, we have

$$
\begin{aligned}
\mathbb{U}^{*} & \left(A^{*} A\right) \mathbb{U} \\
& =\left(\mathbb{U}^{*} A^{*} \mathbb{U}\right)\left(\mathbb{U}^{*} A \mathbb{U}\right) \\
& =\operatorname{diag}\left(\left|\lambda_{11}\right|^{2}, \ldots,\left|\lambda_{1 m}\right|^{2}, \ldots,\left|\lambda_{n 1}\right|^{2}, \ldots,\left|\lambda_{n m}\right|^{2}\right) .
\end{aligned}
$$

Hence, for any $i=1,2, \ldots, n, j=1,2, \ldots, m$, the eigenvalues of matrix $A^{*} A$ are

$$
\lambda_{i j}\left(A^{*} A\right)=\left|\lambda_{i j}\right|^{2}
$$

Therefore, we can get the singular value of $A$ as

$$
\sigma_{i j}(A)=\left[\lambda_{i j}\left(A^{*} A\right)\right]^{1 / 2}=\left|\lambda_{i j}\right| .
$$

Recalling (13) and (14), the proof is completed.

Since the spectral norm of matrix $A$ is defined as

$$
\|A\|_{2}=\max _{\substack{1 \leq i \leq n \\ 1 \leq j \leq m}}\left[\lambda_{i j}\left(A^{*} A\right)\right]^{1 / 2},
$$

by using Theorem 2, we have the following corollary.

Corollary 3. Let $A=B C_{-1,-1}^{n, m}\left(a_{11}, \ldots, a_{1 m}, \ldots, a_{n 1}, \ldots, a_{n m}\right)$ be a block skew circulant matrix with skew circulant blocks; then the spectral norm of matrix $A$ is

$$
\|A\|_{2}=\max _{\substack{1 \leq i \leq n \\ 1 \leq j \leq m}}\left\{\sigma_{i j}\right\} .
$$

By using equations (10) and (11) in [12], we can express the matrix $C_{n \times n}$ and $C_{m \times m}$ as

$$
C_{n \times n}=Q_{n} C_{n 0} Q_{n}^{T}, \quad C_{m \times m}=Q_{m} C_{m 0} Q_{m}^{T},
$$

where

$$
\begin{aligned}
& C_{n 0}=\left(\begin{array}{llll}
C_{11} & & & \\
& C_{22} & & \\
& & \ddots & \\
& & & C_{t t}
\end{array}\right) \text {, } \\
& C_{m 0}=\left(\begin{array}{llll}
D_{11} & & & \\
& D_{22} & & \\
& & \ddots & \\
& & & D_{s s}
\end{array}\right) \text {, } \\
& C_{h h}=\left(\begin{array}{cc}
\cos \theta_{h} & \sin \theta_{h} \\
-\sin \theta_{h} & \cos \theta_{h}
\end{array}\right), \quad h=1,2, \ldots, t . \\
& D_{r r}=\left(\begin{array}{cc}
\cos \theta_{r} & \sin \theta_{r} \\
-\sin \theta_{r} & \cos \theta_{r}
\end{array}\right), \quad r=1,2, \ldots, s .
\end{aligned}
$$

$$
t= \begin{cases}\frac{n}{2}, & \text { when } n \text { is even, } \\ \frac{n-1}{2}+1, & \text { when } n \text { is odd, }\end{cases}
$$

$$
s= \begin{cases}\frac{m}{2}, & \text { when } m \text { is even, } \\ \frac{m-1}{2}+1, & \text { when } m \text { is odd }\end{cases}
$$

So, we can get

$$
A=\mathbb{Q}\left(\sum_{k=1}^{n} \sum_{l=1}^{m} a_{k l} C_{n 0}^{k-1} \otimes C_{m 0}^{l-1}\right) \mathbb{Q}^{T},
$$

where $\mathbb{Q}=\left(Q_{n} \otimes I_{m}\right)\left(I_{n} \otimes Q_{m}\right)$

$$
\sum_{k=1}^{n} \sum_{l=1}^{m} a_{k l} C_{n 0}^{k-1} \otimes C_{m 0}^{l-1}=\left(\begin{array}{ccc}
B_{11} & & \\
& \ddots & \\
& & B_{t t}
\end{array}\right)
$$

and $B_{p p}=\sum_{k=1}^{n} \sum_{l=1}^{m} a_{k l} C_{p p} \otimes C_{m 0}^{l-1}, p=1,2, \ldots, t$.

Let $\Delta A, \Delta b$ be the perturbation of the coefficient matrix $A$ and vector $b$, respectively, where $\Delta A=\operatorname{BC}_{-1,-1}^{n, m}\left(\delta a_{11}, \ldots\right.$, $\left.\delta a_{1 m}, \ldots, \delta a_{n 1}, \ldots, \delta a_{n m}\right)$ is a block skew circulant matrix with skew circulant blocks, has the form as follows:

$$
\begin{aligned}
& \Delta A=\left(\begin{array}{cccc}
\Delta A_{1} & \cdots & \Delta A_{n-1} & \Delta A_{n} \\
-\Delta A_{n} & \Delta A_{1} & \cdots & \Delta A_{n-1} \\
\vdots & \ddots & \ddots & \vdots \\
-\Delta A_{2} & \cdots & -\Delta \dot{A}_{n} & \Delta A_{1}
\end{array}\right) \\
& \Delta A_{k}=\left(\begin{array}{cccc}
\delta a_{k 1} & \cdots & \delta a_{k(m-1)} & \delta a_{k m} \\
-\delta a_{k n} & \delta a_{k 1} & \cdots & \delta a_{k(m-1)} \\
\vdots & \ddots & \ddots & \vdots \\
-\delta a_{k 2} & \cdots & -\delta a_{k m} & \delta a_{k 1}
\end{array}\right) \text {, } \\
& k=1,2, \ldots, n \text {. }
\end{aligned}
$$


Let

$$
\begin{gathered}
\widehat{A}=A+\Delta A, \quad \widehat{b}=b+\Delta b, \\
\widehat{f}\left(\varepsilon_{i}, \delta_{j}\right)=\sum_{k=1}^{n} \sum_{l=1}^{m}\left(a_{k l}+\delta a_{k l}\right) \varepsilon_{i}^{k-1} \delta_{j}^{l-1} .
\end{gathered}
$$

If

$$
\sum_{k=1}^{n} \sum_{l=1}^{m}\left|\delta a_{k l}\right|<\min _{\substack{1 \leq i \leq n \\ 1 \leq j \leq m}}\left\{\sigma_{i j}\right\}
$$

then

$$
\begin{aligned}
\left|\widehat{f}\left(\varepsilon_{i}, \delta_{j}\right)\right|= & \left|\sum_{k=1}^{n} \sum_{l=1}^{m}\left(a_{k l}+\delta a_{k l}\right) \varepsilon_{i}^{k-1} \delta_{j}^{k-1}\right| \\
\geq & \left|\sum_{k=1}^{n} \sum_{l=1}^{m} a_{k l} \varepsilon_{i}^{k-1} \delta_{j}^{l-1}\right| \\
& -\sum_{k=1}^{n} \sum_{l=1}^{m}\left|\delta a_{k l}\right|\left|\varepsilon_{i}\right|^{k-1}\left|\delta_{j}\right|^{l-1} \\
\geq & \min _{\substack{1 \leq i \leq n \\
1 \leq j \leq m}}\left\{\sigma_{i j}\right\}-\sum_{k=1}^{n} \sum_{l=1}^{m}\left|\delta a_{k l}\right|>0 .
\end{aligned}
$$

By using Lemma 1 , we know that $\widehat{A}$ is an invertible matrix. Let

$$
\sigma_{\min }=\min _{\substack{1 \leq j \leq n \\ 1 \leq j \leq m}}\left\{\sigma_{i j}\right\}, \quad \Delta=\sum_{k=1}^{n} \sum_{l=1}^{m}\left|\delta a_{k l}\right| .
$$

By $A x=b, \widehat{A} \widehat{x}=\widehat{b}$, we get

$$
\begin{aligned}
& \widehat{x}-x=\widehat{A}^{-1} \widehat{b}-A^{-1} b \\
&=\widehat{A}^{-1}(b+\delta b)-A^{-1} b \\
&=\widehat{A}^{-1} \delta b+\left(\widehat{A}^{-1}-A^{-1}\right) b \\
&=\widehat{A}^{-1} \delta b+\left(\widehat{A}^{-1}-A^{-1}\right) A x \\
&=\widehat{A}^{-1} \delta b+\widehat{A}^{-1}(A-\widehat{A}) x \\
&\|\widehat{x}-x\|_{2} \leq\left\|\widehat{A}^{-1}\right\|_{2}\|\delta b\|_{2}+\left\|\widehat{A}^{-1}\right\|_{2}\|\widehat{A}-A\|_{2}\|x\|_{2} .
\end{aligned}
$$

Since $\widehat{A}^{-1} \cdot \widehat{A}=I_{m n}$ and $\left\|I_{m n}\right\|_{2}=1$, so we have $\left\|\widehat{A}^{-1}\right\|_{2}\|\widehat{A}\|_{2} \leq 1$. Besides, we know that

$$
\|\widehat{A}\|_{2}=\max _{\substack{1 \leq i \leq n \\ 1 \leq j \leq m}}\left|\widehat{f}\left(\varepsilon_{i}, \delta_{j}\right)\right| \geq \sigma_{\min }-\Delta>0 .
$$

So, we obtain

$$
\left\|\widehat{A}^{-1}\right\|_{2} \leq \frac{1}{\|\widehat{A}\|_{2}} \leq \frac{1}{\sigma_{\min }-\Delta} .
$$

Hence,

$$
\begin{aligned}
\|\widehat{x}-x\|_{2} & \leq \frac{\|\delta b\|_{2}}{\sigma_{\min }-\Delta}+\frac{\|\widehat{A}-A\|_{2}\|x\|_{2}}{\sigma_{\min }-\Delta}, \\
\frac{\|\widehat{x}-x\|_{2}}{\|x\|_{2}} & \leq \frac{\|\delta b\|_{2}}{\left(\sigma_{\min }-\Delta\right)\|x\|_{2}}+\frac{\|\widehat{A}-A\|_{2}}{\sigma_{\min }-\Delta} \\
& =\frac{\|A\|_{2}}{\sigma_{\min }-\Delta}\left[\frac{\|\delta b\|_{2}}{\|A\|_{2}\|x\|_{2}}+\frac{\|\widehat{A}-A\|_{2}}{\|A\|_{2}}\right] \\
& \leq \frac{\|A\|_{2}}{\sigma_{\min }-\Delta}\left[\frac{\|\delta b\|_{2}}{\|b\|_{2}}+\frac{\|\widehat{A}-A\|_{2}}{\|A\|_{2}}\right],
\end{aligned}
$$

where

$$
\|A\|_{2}=\max _{\substack{1 \leq i \leq n \\ 1 \leq j \leq m}}\left\{\sigma_{i j}\right\}
$$

Notice that $\widehat{A}-A=\Delta A$ is a block skew circulant matrix with skew circulant blocks, and $\|\widehat{A}-A\|_{2}=|-1|\|\widehat{A}-A\|_{2}=$ $\|\widehat{A}-A\|_{2}$. So, we get

$$
\begin{aligned}
\|\widehat{A}-A\|_{2} & =\max _{1 \leq j \leq n}\left|\sum_{1 \leq j \leq m}^{n} \sum_{k=1}^{m} \delta a_{k l} \varepsilon_{i}^{k-1} \delta_{j}^{l-1}\right| \\
& \leq \max _{\substack{1 \leq j \leq n \\
1 \leq j \leq m}} \sum_{k=1}^{n} \sum_{l=1}^{m}\left|\delta a_{k l}\right|\left|\varepsilon_{i}\right|^{k-1}\left|\delta_{j}\right|^{l-1} \\
& =\sum_{k=1}^{n} \sum_{l=1}^{m}\left|\delta a_{k l}\right|=\Delta .
\end{aligned}
$$

Hence, we have the following theorem.

Theorem 4. Let $A, \widehat{A}, \delta b, \Delta, \sigma_{\min }$ be defined as above. If $\Delta<$ $\sigma_{\min }$, then

$$
\frac{\|\hat{x}-x\|_{2}}{\|x\|_{2}} \leq \frac{\sigma_{\max }}{\sigma_{\min }-\Delta}\left(\frac{\|\delta b\|_{2}}{\|b\|_{2}}+\frac{\Delta}{\sigma_{\max }}\right),
$$

where

$$
\sigma_{\max }=\|A\|_{2} .
$$

Remark 5. From (38) and (39), the condition number $\mathscr{K}$ of the block skew circulant linear system with skew circulant blocks can be easily computed, as well as the bound of perturbation (38).

3.2. Optimal Backward Perturbation Bound of the Block Skew Circulant Linear System with Skew Circulant Blocks. Let $\widehat{x}$ be an approximate solution to $A x=b$ and let

$$
\Omega \equiv\{(\Delta A, \Delta b) \mid(A+\Delta A) \hat{x}=b+\Delta b\},
$$

$$
\eta(\widehat{x}) \equiv \inf _{(\Delta A, \Delta b) \in \Omega}\|\Delta A, \Delta b\|,
$$

$$
(A+\Delta A) \hat{x}=b+\Delta b,
$$


which is equivalent to

$$
(\Delta A, \Delta b)\left(\begin{array}{c}
\widehat{x} \\
-1
\end{array}\right)=b-A \widehat{x}
$$

Due to [14], we have

$$
\eta(\widehat{x})=\frac{\|b-A \widehat{x}\|_{2}}{\sqrt{1+\|\widehat{x}\|_{2}^{2}}}
$$

( | | | being any unitary invariant norm) .

Let $\widehat{x}$ be an approximate solution to $A x=b$, where $A$ is defined in (1), as follows:

$$
\Omega \equiv\{(\Delta A, \Delta b) \mid(A+\Delta A) \hat{x}=b+\Delta b,
$$

$\Delta A$ is a block skew circulant matrix

with skew circulant blocks\}

$$
\eta(\widehat{x}) \equiv \inf _{(\Delta A, \Delta b) \in \Omega}\left\{\|\Delta A, \Delta b\|_{F}\right\} .
$$

Then $\Omega \neq \phi$ (such as $\Delta A=0$ is a block skew circulant matrix with skew circulant blocks, $\Delta b=A \widehat{x}-b$ )

$$
\eta^{2}(\widehat{x})=\inf _{(\Delta A, \Delta b) \in \Omega}\left\{\|\Delta A\|_{F}^{2}+\|\Delta A \widehat{x}+A \widehat{x}-b\|_{F}^{2}\right\} .
$$

Since

$$
\begin{gathered}
\|\Delta A\|_{F}^{2}=m n \sum_{k=1}^{n} \sum_{l=1}^{m}\left(\delta a_{k l}\right)^{2}, \\
\Delta A=\mathbb{Q}\left(\sum_{k=1}^{n} \sum_{l=1}^{m} \delta a_{k l} C_{n 0}^{k-1} \otimes C_{m 0}^{l-1}\right) \mathbb{Q}^{T},
\end{gathered}
$$

so

$$
\begin{aligned}
& \|\Delta A \widehat{x}+A \widehat{x}-b\|_{F}^{2} \\
& =\left\|\mathbb{Q}\left(\begin{array}{ccc}
\delta B_{11} & & \\
& \ddots & \\
& & \delta B_{t t}
\end{array}\right) \mathbb{Q}^{T} \hat{x}+A \hat{x}-b\right\|_{F}^{2} \\
& =\left\|\left(\begin{array}{ccc}
\delta B_{11} & & \\
& \ddots & \\
& & \delta B_{t t}
\end{array}\right)\left(\begin{array}{c}
x_{1}^{(0)} \\
\vdots \\
x_{t}^{(0)}
\end{array}\right)-r_{0}\right\|_{F}^{2} \\
& =\left\|\left(\begin{array}{c}
\left(\sum_{k=1}^{n} \sum_{l=1}^{m} \delta a_{k l} C_{11}^{k-1} \otimes C_{m 0}^{l-1}\right) x_{1}^{(0)} \\
\vdots \\
\left(\sum_{k=1}^{n} \sum_{l=1}^{m} \delta a_{k l} C_{t t}^{k-1} \otimes C_{m 0}^{l-1}\right) x_{t}^{(0)}
\end{array}\right)-r_{0}\right\|_{F}^{2} \\
& =\left\|G_{0}\left(\delta a_{11}, \ldots, \delta a_{1 m}, \ldots, \delta a_{n 1}, \ldots, \delta a_{n m}\right)^{T}-r_{0}\right\|_{F}^{2},
\end{aligned}
$$

$p=1,2, \ldots, t, k=1,2, \ldots, n$, and $l=1,2, \ldots, m$

Let

$$
\begin{aligned}
& f\left(\delta a_{11}, \ldots, \delta a_{n m}\right) \\
& \quad=m n \sum_{k=1}^{n} \sum_{l=1}^{m}\left(\delta a_{k l}\right)^{2}+\left\|G_{0}\left(\begin{array}{c}
\delta a_{11} \\
\vdots \\
\delta a_{n m}
\end{array}\right)-r_{0}\right\|_{F}^{2},
\end{aligned}
$$

then

$$
\frac{\partial f}{\partial \delta a_{k l}}=0
$$

which is equivalent to

$$
\begin{gathered}
\left(2 m n I_{m n}+2 G_{0}^{T} G_{0}\right)\left(\begin{array}{c}
\delta a_{11} \\
\vdots \\
\delta a_{n m}
\end{array}\right)-2 G_{0}^{T} r_{0}=0, \\
\frac{\partial^{2} f}{\partial\left(\delta a_{k l}\right)^{2}}=2 m n I_{m n}+2 G_{0}^{T} G_{0}>0 .
\end{gathered}
$$

Hence $f$ is a convex function about $\left(\delta a_{11}, \ldots, \delta a_{n m}\right)$, and the point of minimal value is

$$
\left(\begin{array}{c}
\delta a_{11} \\
\vdots \\
\delta a_{n m}
\end{array}\right)=\left(m n I_{m n}+G_{0}^{T} G_{0}\right)^{-1} G_{0}^{T} r_{0}
$$

Substituting it into (48), we can get the following.

Theorem 6. Let $r_{0}$ and $G_{0}$ be defined as above; then we have

$$
\begin{aligned}
\eta(\widehat{x})^{2}= & m n r_{0}^{T} G_{0}\left(m n I_{m n}+G_{0}^{T} G_{0}\right)^{-2} G_{0}^{T} r_{0} \\
& +\left\|\left[G_{0}\left(m n I_{m n}+G_{0}^{T} G_{0}\right)^{-1} G_{0}^{T}-I_{m n}\right] r_{0}\right\|_{F}^{2} .
\end{aligned}
$$


Let $G_{0}=U \Sigma V^{T}$ be the singular value decomposition of $G_{0}$, where $U$ and $V$ are real orthogonal matrices, $\Sigma=$ $\operatorname{diag}\left(\sigma_{1}^{\prime}, \ldots, \sigma_{n m}^{\prime}\right), \sigma_{j}^{\prime} \geq 0(j=1,2, \ldots, n m)$, so

$$
\begin{aligned}
\eta(\widehat{x})^{2}= & m n r_{0}^{T} U \Sigma V^{T}\left(m n I_{m n}+\Sigma^{2}\right)^{-2} V \Sigma U^{T} r_{0} \\
& +\left\|\left[U \Sigma V^{T}\left(m n I_{m n}+\Sigma^{2}\right)^{-1} V \Sigma U^{T}-I_{m n}\right] r_{0}\right\|_{F}^{2} \\
= & m n r_{1}^{T} \Sigma\left(m n I_{m n}+\Sigma^{2}\right)^{-2} \Sigma r_{1} \\
& +\left\|\left[\Sigma\left(m n I_{m n}+\Sigma^{2}\right)^{-1} \Sigma-I_{m n}\right] r_{0}\right\|_{F}^{2} \\
= & m n r_{1}^{T} \Sigma\left(m n I_{m n}+\Sigma^{2}\right)^{-2} \Sigma r_{1} \\
& +\left\|\left[\Sigma\left(m n I_{m n}+\Sigma^{2}\right)^{-1} \Sigma-I_{m n}\right] r_{1}\right\|_{F}^{2} \\
= & m n r_{1}^{T} \Sigma\left(m n I_{m n}+\Sigma^{2}\right)^{-2} \Sigma r_{1} \\
& +m^{2} n^{2} r_{1}^{T}\left(m n I_{m n}+\Sigma^{2}\right)^{-2} r_{1} \\
= & r_{1}^{T} \operatorname{diag}\left(d_{1}, d_{2}, \ldots, d_{m n}\right) r_{1},
\end{aligned}
$$

where $r_{1}=U^{T} r_{0}, d_{j}=\left(m n \sigma_{j}^{\prime 2}+m^{2} n^{2}\right) /\left(m n+\sigma_{j}^{\prime 2}\right)^{2}=m n /$ $\left(m n+\sigma_{j}^{\prime 2}\right), j=1,2, \ldots, m n$.

Remark 7. By $\sigma_{j}^{2} \leq\left\|G_{0}\right\|_{F}^{2}=m n\|\widehat{x}\|_{2}^{2}$, we get $1+\sigma_{j}^{\prime 2} / m n \leq$ $1+\|\widehat{x}\|_{2}^{2}$, and hence $m n /\left(m n+\sigma_{j}^{\prime 2}\right) \geq 1 /\left(1+\|\widehat{x}\|_{2}^{2}\right)$.

Algorithm 8. Consider the following.

Step 1. Form the block style spectral decomposition of the matrix $C_{n \times n}$ and $C_{m \times m}$,

$$
\begin{gathered}
C_{n \times n}=Q_{n}\left(\begin{array}{cccc}
C_{11} & & & \\
& C_{22} & & \\
& & \ddots & \\
& & & C_{t t}
\end{array}\right) Q_{n}^{T}, \\
C_{m \times m}=Q_{m}\left(\begin{array}{cccc}
D_{11} & & & \\
& D_{22} & & \\
& & \ddots & \\
& & & D_{s s}
\end{array}\right) Q_{m}^{T} .
\end{gathered}
$$

Step 2. Compute $\mathbb{Q}=\left(Q_{n} \otimes I_{m}\right)\left(I_{n} \otimes Q_{m}\right)$.

Step 3. Compute $r=b-A \widehat{x}$.

Step 4. Compute $r_{0}=\mathbb{Q}^{T} r$.

Step 5. Compute $\mathbb{Q}^{T} \widehat{x}=\left(x_{1}^{(0)}, \ldots, x_{t}^{(0)}\right)^{T}$.

Step 6. Form $G_{0}$.

Step 7. Compute $\eta^{2}(\widehat{x})$.
TABLE 1

\begin{tabular}{lcccc}
\hline & $\epsilon$ & $\mathscr{K}$ & $\eta_{1}(\widehat{x})$ & $\eta_{2}(\widehat{x})$ \\
\hline Case 0 & 0 & 6.472 & 0 & 0 \\
Case 1 & 0.0694 & 6.193 & 0.1252 & 0.2487 \\
Case 2 & 0.0075 & 6.438 & 0.0127 & 0.0223 \\
Case 3 & $7.5122 \times 10^{-4}$ & 6.468 & 0.0013 & 0.0022 \\
\hline
\end{tabular}

3.3. Numerical Example. In this section, we give a simple numerical example to verify the conclusions above. Suppose that $n=3, m=2$ in the following example.

If the coefficient matrix of block skew circulant linear system with skew circulant blocks is $A=\mathrm{BC}_{-1,-1}^{3,2}(1,2,4.5$, $3,4,2.5)$, and the constant vector $b=(1,2,1,0,3,4)^{T}$. Now, for comparative analysis, we give three perturbations in the following:

$$
\begin{gathered}
\Delta A_{1}=\mathrm{BC}_{-1,-1}^{3,2}(0.01,0.03,0.02,-0.05,-0.03,0.01), \\
\Delta b_{1}=(0.1,0.3,0,-0.2,0.01,0.04)^{T}, \\
\Delta A_{2}=\mathrm{BC}_{-1,-1}^{3,2}(0.01,0.01,0.01,0.01,0.01,0.01), \\
\Delta b_{2}=(0.01,0.01,0.01,0.01,0.01,0.01)^{T}, \\
\Delta A_{3}=\mathrm{BC}_{-1,-1}^{3,2}(0.001,0.001,0.001,0.001,0.001,0.001), \\
\Delta b_{3}=(0.001,0.001,0.001,0.001,0.001,0.001)^{T},
\end{gathered}
$$

then by equation $\widehat{A} \widehat{x}=\widehat{b}$, we get the approximate solution of $A x=b$ correspondingly as

$$
\begin{aligned}
& x=\left(\begin{array}{c}
-1.0505, \\
0.6490, \\
1.0280, \\
-1.1028, \\
-0.9215, \\
1.2482
\end{array}\right), \quad \widehat{x}_{1}=\left(\begin{array}{c}
-1.1526, \\
0.6358, \\
1.1192, \\
-1.0611, \\
-1.0158, \\
1.2286
\end{array}\right), \\
& \widehat{x}_{2}=\left(\begin{array}{c}
-1.0396, \\
0.6473, \\
1.0178, \\
-1.1003, \\
-0.9109, \\
1.2484
\end{array}\right), \quad \widehat{x}_{3}=\left(\begin{array}{c}
-1.0494, \\
0.6489, \\
1.0270, \\
-1.1025, \\
-0.9204, \\
1.2482
\end{array}\right),
\end{aligned}
$$

where $x$ is the solution of $A x=b$ and $x_{i}(i=1,2,3)$ is the solution of $\left(A+\Delta A_{i}\right) x=b+\Delta b$.

According to the Algorithm, we obtain Table 1, where $\epsilon$ means relative error of block skew circulant linear system with skew circulant blocks, $\mathscr{K}=\max \left\{\sigma_{i j}\right\} / \min \left\{\sigma_{i j}\right\}$ is the condition number, $\eta_{1}(\widehat{x})=\|b-A \widehat{x}\|_{2} / \sqrt{1+\|\widehat{x}\|_{2}^{2}}$ and $\eta_{2}$ are obtained from the Algorithm.

From the tabular, we know that the conclusions above are right and the Algorithm is efficient. 


\section{Conclusion}

The related problems of block skew circulant matrix with skew circulant blocks are considered in this paper. We not only present block style spectral decomposition and singular value, but also study backward perturbation analysis for the block skew circulant linear system with skew circulant blocks. The reason why we focus our attentions on block skew circulant matrix with skew circulant blocks is to explore the application of block skew circulant matrix with skew circulant blocks in the related field of medicine and realtime tracking. On the basis of existing application situation [23], we conjecture that SVD decomposition of block skew circulant matrix with skew circulant blocks will play an important role in CT-perfusion imaging of human brain. On the basis of method [7] and ideas of [24], we will exploit real-time tracking with kernel matrix of block skew circulant matrix with skew circulant blocks structure. The circulant singular value decomposition (cSVD) techniques with a block-circulant deconvolution matrix [25-29] were used to perform the deconvolution calculation to obtain the $[\operatorname{rCBF} \cdot R(t)]$ curve $[25,26]$. The maximum value of the [rCBF. $R(t)]$ curve was used as the rCBF. We will exploit the skew circulant singular value decomposition (scSVD) techniques of a block skew circulant matrix with skew circulant blocks deconvolution matrix to obtain the $[\mathrm{rCBF} . R(t)]$ curve.

\section{Conflict of Interests}

The authors declare that there is no conflict of interests regarding the publication of this paper.

\section{Acknowledgments}

The authors thank the Development Project of Science and Technology of Shandong Province (Grant no. 2012GGX10115) and NSFC (Grant no. 11201212) and the AMEP of Linyi University, China, and the same thanks go to the works by all the authors in the references .

\section{References}

[1] D. Bertaccini and M. K. Ng, "Skew-circulant preconditioners for systems of LMF-based ODE codes," in Numerical Analysis and Its Applications, vol. 1988 of Lecture Notes in Computer Science, pp. 93-101, Springer, Berlin, Germany, 2001.

[2] R. H. Chan and X.-Q. Jin, "Circulant and skew-circulant preconditioners for skew-Hermitian type Toeplitz systems," BIT, vol. 31, no. 4, pp. 632-646, 1991.

[3] R. H. Chan and K.-P. Ng, "Toeplitz preconditioners for Hermitian Toeplitz systems," Linear Algebra and Its Applications, vol. 190, pp. 181-208, 1993.

[4] T. Huckle, "Circulant and skewcirculant matrices for solving Toeplitz matrix problems," SIAM Journal on Matrix Analysis and Applications, vol. 13, no. 3, pp. 767-777, 1992.

[5] J. N. Lyness and T. Sörevik, "Four-dimensional lattice rules generated by skew-circulant matrices," Mathematics of Computation, vol. 73, no. 245, pp. 279-295, 2004.
[6] N. Akhondi and F. Toutounian, "Accelerated circulant and skew circulant splitting methods for Hermitian positive definite Toeplitz systems," Advances in Numerical Analysis, vol. 2012, Article ID 973407, 17 pages, 2012.

[7] M. J. Narasimha, "Linear convolution using skew-cyclic convolutions," IEEE Signal Processing Letters, vol. 14, no. 3, pp. 173-176, 2007.

[8] D. Q. Fu, Z. L. Jiang, Y. F. Cui, and S. T. Jhang, "A new fastalgorithm for optimal design of block digital filters by skewcyclic convolution," IET Signal Processing, 6 pages, 2014.

[9] V. C. Liu and P. P. Vaidyanathan, "Circulant and skew-circulant matrices as new normal-form realization of IIR digital filters," IEEE Transactions on Circuits and Systems, vol. 35, no. 6, pp. 625-635, 1988.

[10] T. M. Foltz and B. M. Welsh, "Symmetric convolution of asymmetric multidimensional sequences using discrete trigonometric transforms," IEEE Transactions on Image Processing, vol. 8, no. 5, pp. 640-651, 1999.

[11] X. Liu and X. Guo, "On optimal backward perturbation analysis for the linear system with block cyclic coefficient matrix," Numerical Mathematics, vol. 12, no. 2, pp. 162-172, 2003.

[12] J. Li, Z. Jiang, N. Shen, and J. Zhou, "On optimal backward perturbation analysis for the linear system with skew circulant coefficient matrix," Computational and Mathematical Methods in Medicine, vol. 2013, Article ID 707381, 7 pages, 2013.

[13] J.-G. Sun and Z. Sun, "Optimal backward perturbation bounds for underdetermined systems," SIAM Journal on Matrix Analysis and Applications, vol. 18, no. 2, pp. 393-402, 1997.

[14] J.-L. Rigal and J. Gaches, "On the compatibility of a given solution with the data of a linear system," Journal of the Association for Computing Machinery, vol. 14, pp. 543-548, 1967.

[15] A. Beck, Y. C. Eldar, and A. Ben-Tal, "Minimax mean-squared error estimation of multichannel signals," IEEE Transactions on Information Theory. In press.

[16] A. Beck, A. Ben-Tal, and Y. C. Eldar, "Robust mean-squared error estimation of multiple signals in linear systems affected by model and noise uncertainties," Mathematical Programming, vol. 107, no. 1-2, pp. 155-187, 2006.

[17] A. K. Katsaggelos, K. T. Lay, and N. P. Galatsanos, "General framework for frequency domain multi-channel signal processing," IEEE Transactions on Image Processing, vol. 2, no. 3, pp. 417-420, 1993.

[18] H. Murakami, "Discrete wavelet transform based on cyclic convolutions," IEEE Transactions on Signal Processing, vol. 52, no. 1, pp. 165-174, 2004.

[19] M. Sznaier, O. Camps, and C. Mazzaro, "Finite horizon model reduction of a class of neutrally stable systems with applications to texture synthesis and recognition," in Proceedings of the 43rd IEEE Conference on Decision and Control (CDC '04), pp. 30683073, December 2004.

[20] Z. L. Jiang and Z. X. Zhou, Circulant Matrices, Chengdu Technology University, Chengdu, China, 1999.

[21] Z. L. Jiang and Y. R. Guo, "Nonsingularity on level-2 $\left(r_{1}, r_{2}\right)$ circulant matrices of type $(m, n)$," Chinese Quarterly Journal of Mathematical, vol. 11, no. 2, pp. 106-110, 1996.

[22] R. A. Horn and C. R. Johnson, Matrix Analysis, Posts \& Telecom Press, 2005.

[23] H.-J. Wittsack, A. M. Wohlschläger, E. K. Ritzl et al., "CTperfusion imaging of the human brain: advanced deconvolution analysis using circulant singular value decomposition," Computerized Medical Imaging and Graphics, vol. 32, no. 1, pp. 67-77, 2008. 
[24] J. F. Henriques, R. Caseiro, P. Martins, and J. Batista, "Exploiting the circulant structure of tracking-by-detection with kernels," in Proceedings of the European Conference on Computer Vision (ECCV'12), pp. 1-14, 2012.

[25] L. Østergaard, R. M. Weisskoff, D. A. Chesler, G. Gyldensted, and B. R. Rosen, "High resolution measurement of cerebral blood flow using intravascular tracer bolus passages. Part I: Mathematical approach and statistical analysis," Magnetic Resonance in Medicine, vol. 36, no. 5, pp. 715-725, 1996.

[26] L. Østergaard, A. G. Sorensen, K. K. Kwong, R. M. Weisskoff, C. Gyldensted, and B. R. Rosen, "High resolution measurement of cerebral blood flow using intravascular tracer bolus passages. Part II: Experimental comparison and preliminary results," Magnetic Resonance in Medicine, vol. 36, no. 5, pp. 726-736, 1996.

[27] O. Wu, L. Østergaard, R. M. Weisskoff, T. Benner, B. R. Rosen, and A. G. Sorensen, "Tracer arrival timing-insensitive technique for estimating flow in MR perfusion-weighted imaging using singular value decomposition with a block-circulant deconvolution matrix," Magnetic Resonance in Medicine, vol. 50, no. 1, pp. 164-174, 2003.

[28] O. Wu, L. Østergaard, W. J. Koroshetz et al., "Effects of tracer arrival time on flow estimates in MR perfusion-weighted imaging," Magnetic Resonance in Medicine, vol. 50, no. 4, pp. 856-864, 2003.

[29] M. M. H. Teng, I.-C. Cho, Y.-H. Kao, C.-S. Chuang, F.-Y. Chiu, and F.-C. Chang, "Improvements in the quantitative assessment of cerebral blood volume and flow with the removal of vessel voxels from MR perfusion images," BioMed Research International, vol. 2013, Article ID 382027, 11 pages, 2013. 


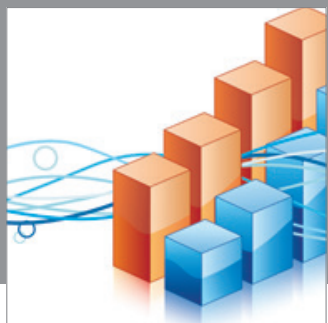

Advances in

Operations Research

mansans

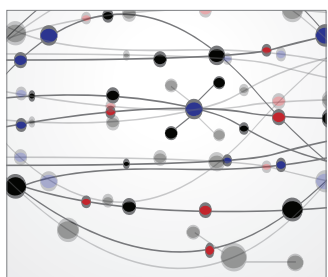

The Scientific World Journal
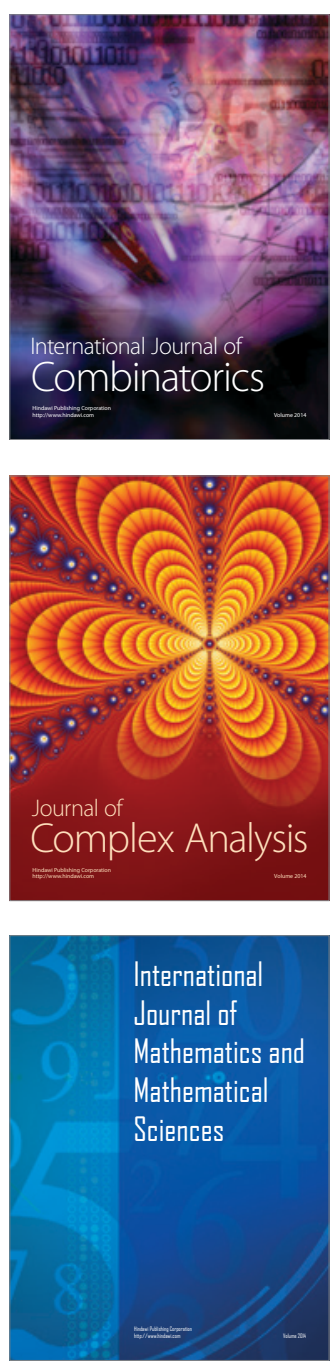
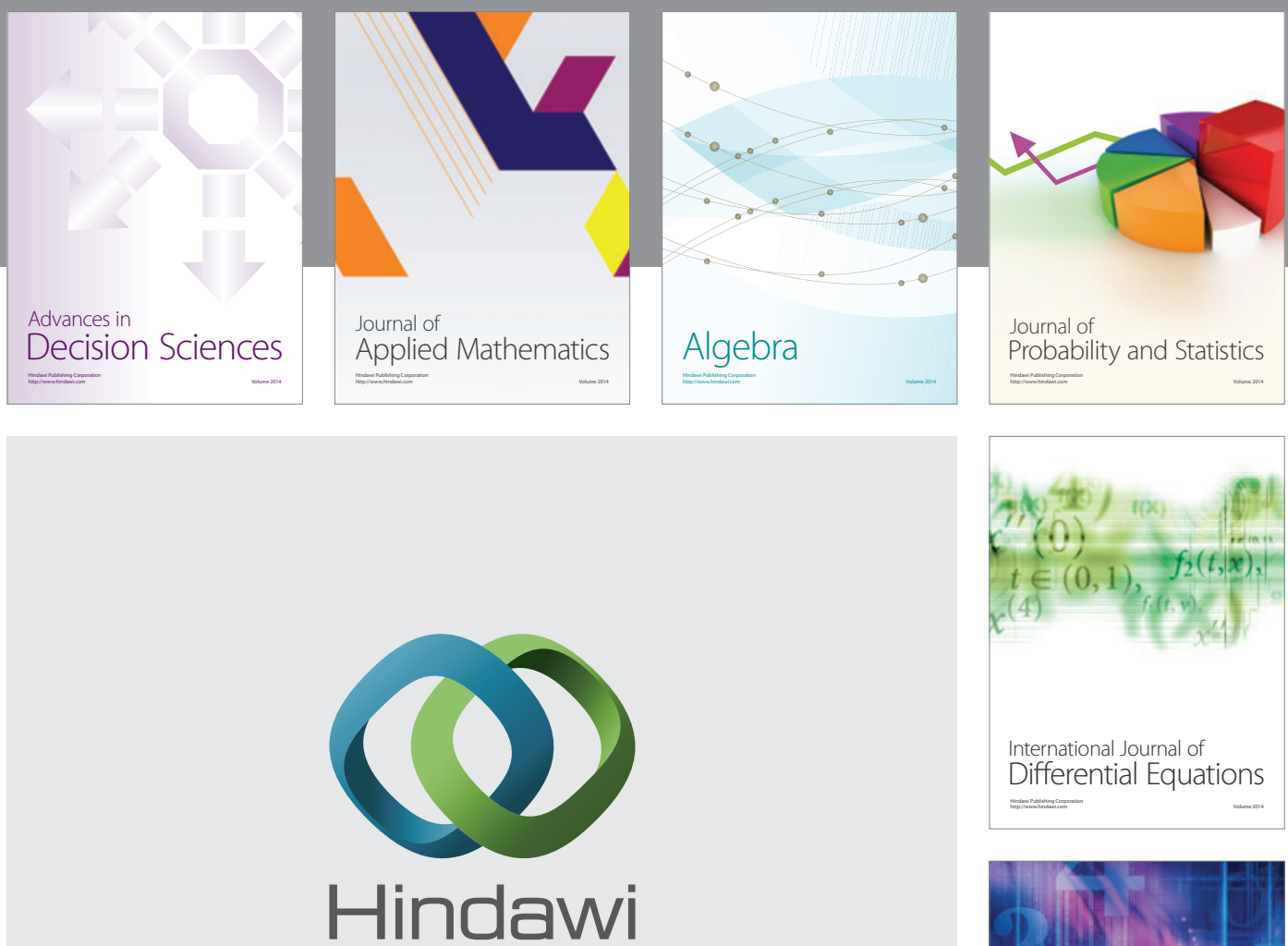

Submit your manuscripts at http://www.hindawi.com
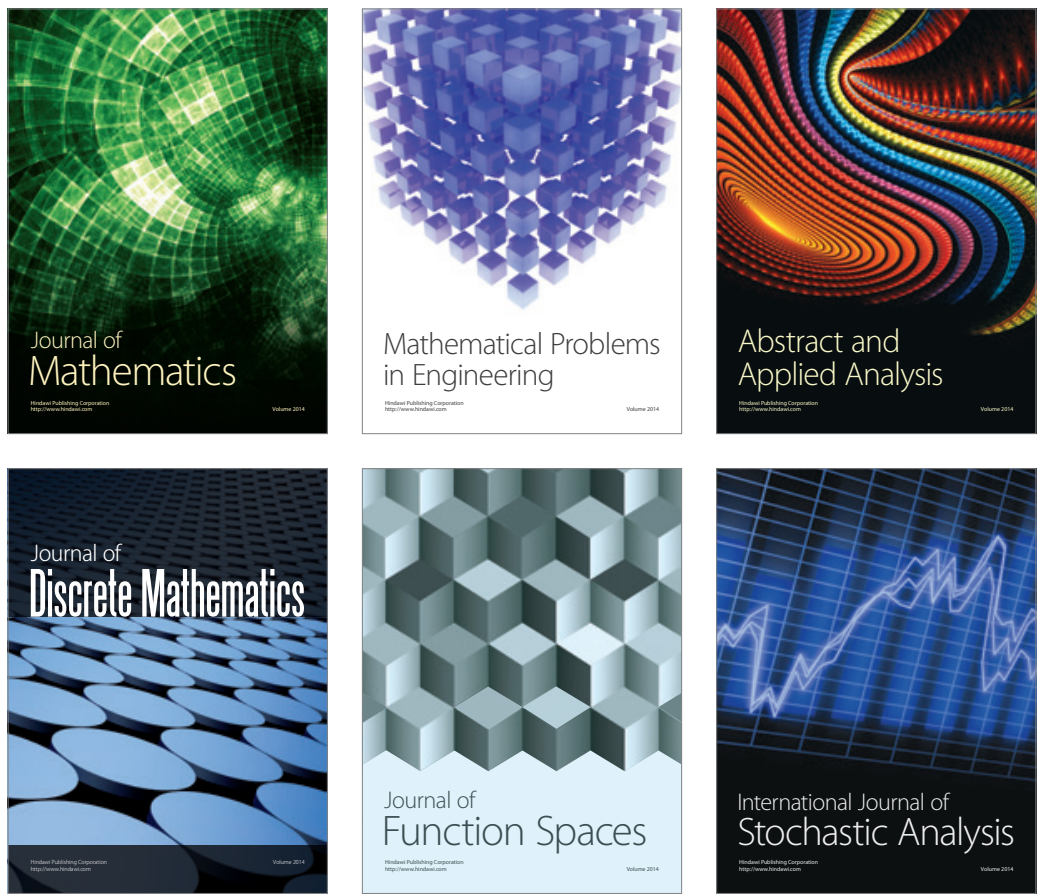

Journal of

Function Spaces

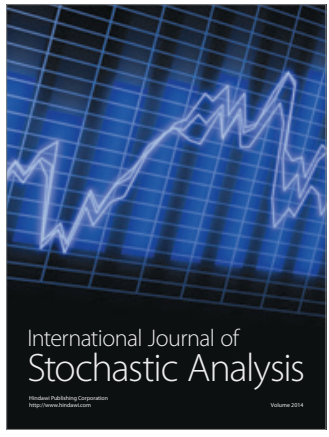

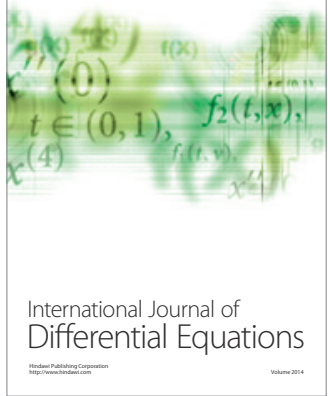
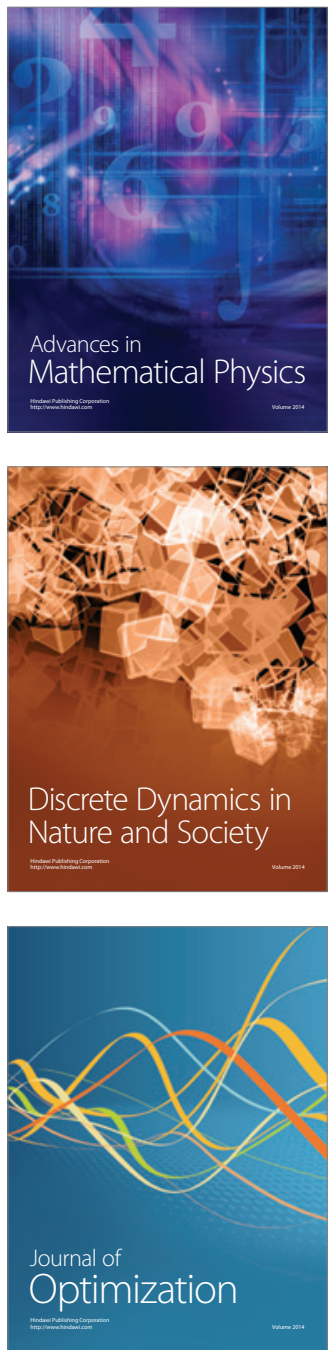